\title{
Simulation Analysis of Silicon Ingot Growth in Directional Solidification System
}

\author{
Jun Dai, ${ }^{1}$ Yao-Chung Yang, ${ }^{2}$ Chao-Ming Hsu, ${ }^{2 *}$ \\ Hsien-Wei Tseng, ${ }^{1}$ Peng Wang, ${ }^{1}$ and Cheng-Fu Yang $3{ }^{3, * *}$ \\ ${ }^{1}$ College of Artificial Intelligence, Yango University, Mawei District, Fujian 350015, China \\ ${ }^{2}$ Department of Mechanical Engineering, National Kaohsiung University of Applied Science, \\ Kaohsiung 807, Taiwan \\ ${ }^{3}$ Department of Chemical and Materials Engineering, National University of Kaohsiung, Kaohsiung 811, Taiwan \\ ${ }^{4}$ Department of Aeronautical Engineering, Chaoyang University of Technology, Taichung 413, Taiwan
}

(Received March 23, 2021; accepted June 14, 2021)

Keywords: simulation, directional solidification, numerical analysis, polycrystalline silicon

Polycrystalline silicon wafers are the main sources of solar cells. In this study, we used numerical analysis to optimize the growth of polycrystalline silicon ingots in a directional solidification system (DSS). The numerical analysis method was based on the finite volume method (FVM), using Fluent software as a tool to analyze the thermal field of the directional solidification process through the solidified-molten model, considering the effects of heat conduction, heat convection, and heat radiation. We used a GT Solar Ltd. directional solidification crystal growth furnace as the prototype for the finite element model analysis. We used triangles and quadrilaterals as the cutting meshes to simulate the two-dimensional structure using Fluent. The software and operating processes were divided into three processes: pretreatment, numerical calculation, and postprocessing. We used the simulation technology to understand the effect of the temperature profile on the growth characteristics of polycrystalline silicon wafers during the silicon ingot growth process, allowing us to optimize the crystal growth process.

\section{Introduction}

Recently, more domestic and foreign researchers and companies have focused their research on the growth of polycrystalline silicon. The investigation has focused on how to reduce the impurity concentration and enhance the growth rate and on analyzing the distribution of the thermal field in the growth furnace ${ }^{(1-5)}$ with the aim of growing silicon ingots with higher solar photovoltaic conversion efficiency that can be used to fabricate solar cells with greater costeffectiveness. A directional solidification system (DSS) is mainly used to manufacture and produce polycrystalline silicon, and most manufacturers use the DSS furnace produced by GT Solar Ltd. The DSS furnace allows axial heat conduction and minimizes heat loss in the horizontal direction, enabling the silicon ingot to grow in one direction. Because the one-round growth of a polycrystalline silicon ingot takes a long time, making its experimental investigation

\footnotetext{
*Corresponding author: e-mail: jammy@kuas.edu.tw

** Corresponding author: e-mail: cfyang@nuk.edu.tw

https://doi.org/10.18494/SAM.2021.3386
} 
time-consuming, we have developed a numerical analysis method to simulate and analyze the distribution of the thermal field in the growth furnace. We improved the internal structure of a furnace and changed the state of the temperature field to obtain the optimal growth parameters. ${ }^{(4-8)}$ We analyzed the internal temperature field distribution and discussed the ingot growth process in the DSS furnace. We compared the results obtained from numerical analysis and an actual experiment and improved the growth process. When the simulation technology is used to find the optimum parameters, less labor, materials, and time are required for the manufacture of silicon ingots, and the simulation parameters of the ingot growth furnace can be changed arbitrarily without adding to the cost.

Numerical analysis technologies are widely used to simulate the cooling rate of silicon ingots, and computational fluid dynamics (CFD) is now one of the most widely used technologies in the analysis of fluid mechanics. The finite volume method (FVM) is a discrete technology that has rapidly developed in recent years. The FVM has high computational efficiency and is widely used in the CFD field. Many CFD software packages have been developed to use the FVM, for example, Fluent, ANSYS CFX, and STAR-CD. Fluent is one of the most widely used CFD software packages because it can simulate and analyze the fluid flow and heat transfer in complicated geometric areas. Fluent allows the use of flexible mesh characteristics by supporting many different mesh structures; thus, users can freely choose unstructured or structured meshes to divide the geometric area. For example, triangle and quadrilateral meshes can be used to simulate two-dimensional structures, and tetrahedron, hexahedron, cuneiform, orthorhombic pyramid, and polyhedron meshes can be used to simulate three-dimensional structures. Fluent can also support the use of mixed meshes and has a user-friendly interface that is easy for new users to use. Fluent was designed on the basis of the thinking of a CFD software group, i.e., from the perspective of users. Using Fluent, we can focus on the physical phenomena of complex fluid fields, because this software has the functions of different discrete grids and specialist numerical methods to obtain the best combination of calculation speed, stability, and accuracy. Fluent can be used to solve complex fluid calculation problems in different research fields with high efficiency.

Silicon ingots are used as semiconductors in many applications and fields, including the chemical and physical sensors used in genetic diagnostics, pollution control, medicine, biophysics, biochemistry, asthma diagnosis, drug delivery, optical switching, and optical sensors. $.9,10)$ Therefore, the manufacture of high-quality ingots for the substrates of sensors is very important. In this study, we examined silicon ingot growth in a DSS furnace. ${ }^{(6,11-14)}$ Next, we used a numerical analysis method to find the effects of different parameters on the solidliquid phase distribution map in the silicon ingot growth process in a DSS furnace, including the geometric model, the meshes, the governing equations, and the calculation processes and boundary conditions of the used algorithm.

\section{Simulation Parameters}

The simulation analysis of silicon ingot growth in a DSS furnace using software was divided into three processes: pretreatment, numerical calculation, and postprocessing. 
1. Pretreatment: we used Autodesk computer-aided design (AutoCAD) software to construct and lay out the two-dimensional model and used the design modeler to repair the model, specify boundaries, and finally grid the meshes.

2. Numerical calculation: we used Fluent to perform the numerical calculation, along with a visual studio functional library to designate the user's defined functions.

3. Postprocessing: we imported the results calculated using Fluent to perform the data processing of the tabular ribbon distribution map, contour distribution map, and point data.

The silicon ingot growth involves five processes: heating, melting, growth, annealing, and cooling. Figure 1(a) shows the two-dimensional layout of the main parts and Fig. 1(b) shows the work flow field of the silicon ingot growth furnace manufactured by GT Solar Ltd.; we used this DSS furnace for the simulation of heating fields. The temperature in the ingot growth furnace was controlled by the heater, and there were two temperature sensors, TC1 and TC2. TC1 was used to monitor the temperature of the heater, and TC2 was used to monitor the temperature of the graphite protection cage. A water-cooling system was included in the furnace wall, and the temperature outside the furnace was kept at room temperature. We used AutoCAD to construct a two-dimensional global-model mesh, as shown in Fig. 1(b).

Skewness was used to evaluate the mesh quality and is defined as

$$
\text { Skewness }=\frac{G-H}{G},
$$

where $G$ is the optimal cell size and $H$ is the cell size. Skewness has a value between 0 and 1 . When the skewness is 0 , the mesh has the optimal characteristic and consists of regular polygons (equilateral triangles or squares). A skewness of 1 suggests that the differences between the

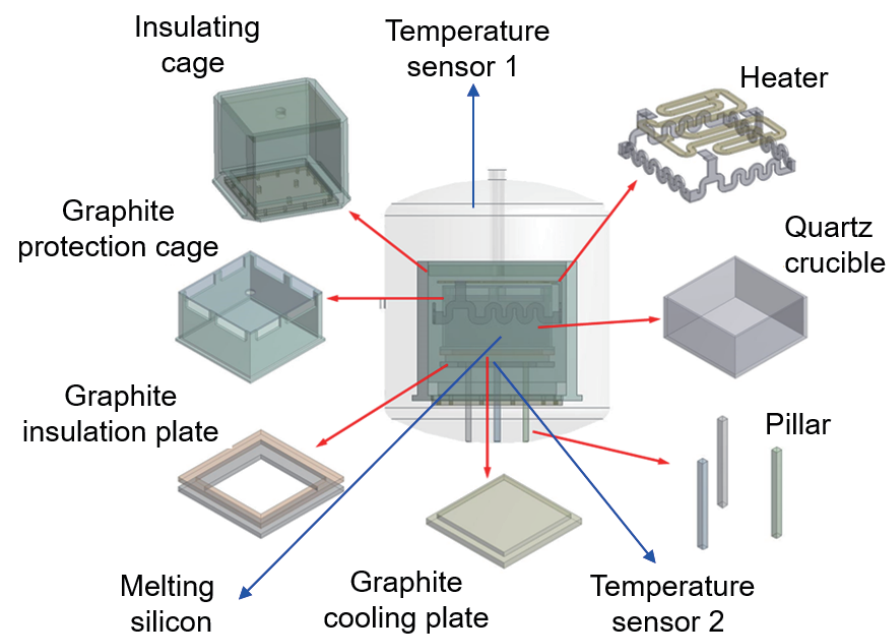

(a)

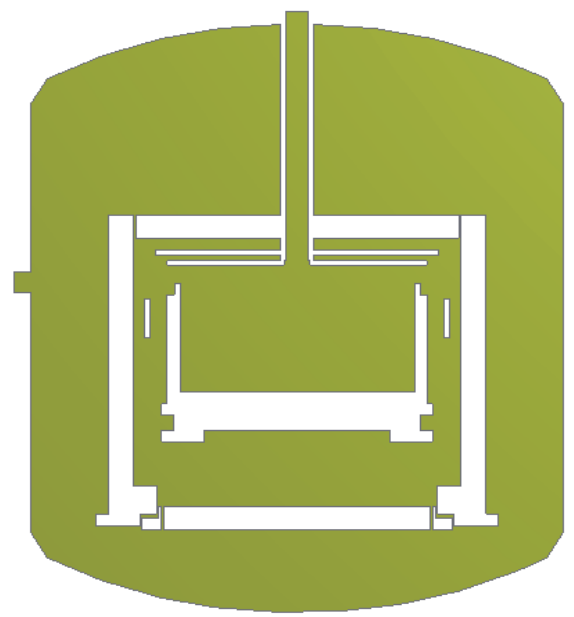

(b)

Fig. 1. (Color online) (a) Two-dimensional layout of main parts and (b) work flow field of silicon ingot growth furnace. 
maximum and minimum angles of the polygon meshes is too large. Table 1 shows the skewness values for different qualities of the mesh. The default setting of Fluent is that when the skewness value is larger than 0.97 , the calculation process is terminated. However, the skewness of the initial meshes and remeshes must be controlled to less than 0.97 , and the smaller the skewness, the better.

The insulation cage was divided into movable and unmovable regions, as shown in Fig. 2. The unmovable region used triangle unstructured meshes, allowing the region to go through the remeshing process. There are two remeshing mechanisms: one evaluates the length of the meshes and the other evaluates the skewness. When the length of one mesh was smaller than $6.5 \mathrm{~mm}$, it was combined with neighboring meshes, and when the length of one mesh was larger than $11 \mathrm{~mm}$, it was split into two meshes. When the skewness of one mesh was larger than 0.6 , it was combined with neighboring meshes or split to reduce the skewness to less than 0.6. The other parts and the fluid field were constructed using quadrilateral structured meshes. Figure 3 shows schematic diagrams of the initial mesh and the remeshes after different times.

We first compared the simulated numerical calculation results of the DSS ingot furnace with the actual results of the fabrication process. The simulated results were compared with the temperature extracted from the TC2 temperature sensor. Also, the temperature changes were

Table 1

Range of skewness values for different mesh qualities.

\begin{tabular}{lc}
\hline Skewness & Quality \\
\hline 1 & Poorest (acute angle is too large) \\
$0.9-<1$ & Poor \\
$0.75-0.9$ & Unsatisfactory \\
$0.5-0.75$ & General \\
$0.25-0.5$ & Good \\
$>0-0.25$ & Excellent \\
0 & Optimal (equiangular) \\
\hline
\end{tabular}

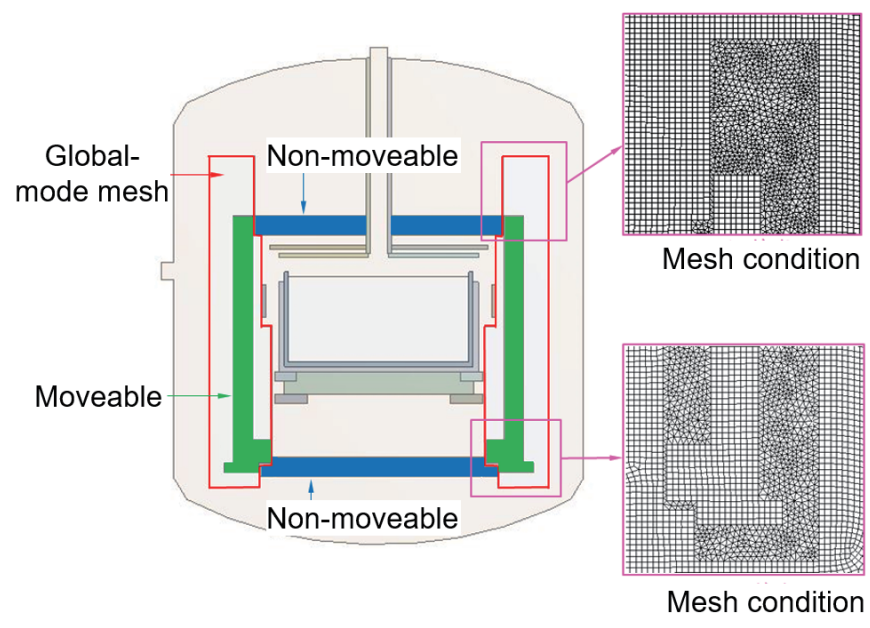

Fig. 2. (Color online) Schematic diagrams of insulation cage. 


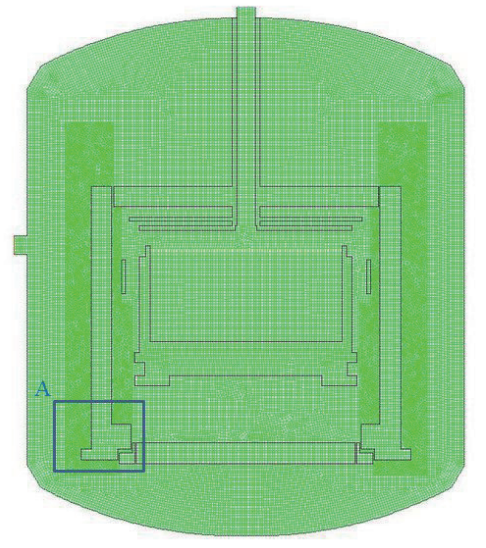

(a)

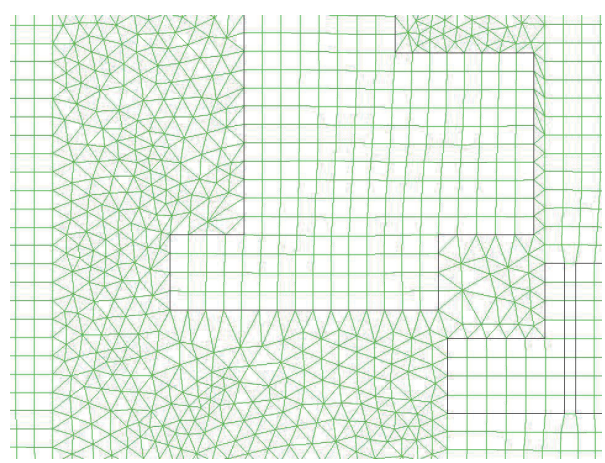

(c)

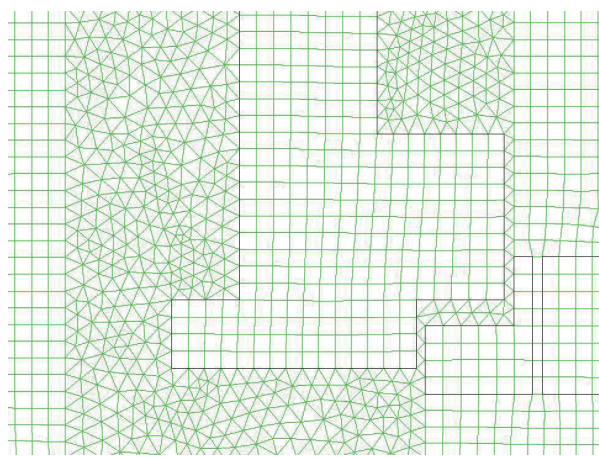

(b)

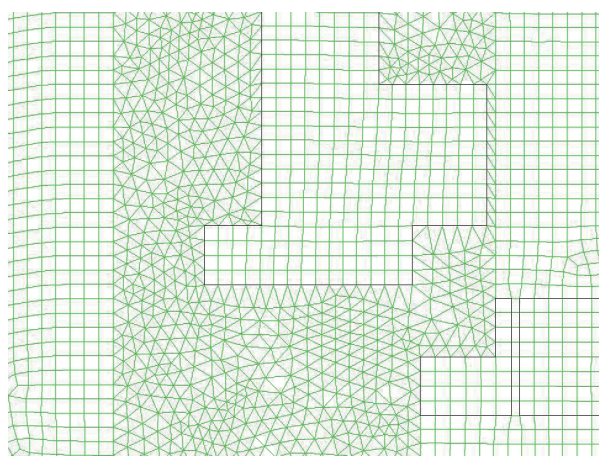

(d)

Fig. 3. (Color online) Meshes after different times. (a) Whole structure, (b) local state after $0.5 \mathrm{~h}$, (c) local state after $1 \mathrm{~h}$, and (d) local state after $20 \mathrm{~h}$.

observed at six positions in the melted silicon: points $\mathrm{A}, \mathrm{B}$, and $\mathrm{C}$ were 30,200 , and $370 \mathrm{~mm}$ from the bottom of the furnace, and points $\mathrm{A}^{*}, \mathrm{~B}^{*}$, and $\mathrm{C}^{*}$ were located $390 \mathrm{~mm}$ to the left of points $\mathrm{A}, \mathrm{B}$, and $\mathrm{C}$, respectively ( $30 \mathrm{~mm}$ from the crucible wall), as shown in Fig. 4.

\section{Simulation Processes and Results}

In this study, the FVM was used to find the solution of the equations governing the DSS furnace. The governing equations of each control volume are as follows. First, the continuity equation is

$$
\frac{\partial \rho}{\partial t}+\frac{\partial\left(\rho u_{x}\right)}{\partial x}+\frac{\partial\left(\rho u_{y}\right)}{\partial y}+\frac{\partial\left(\rho u_{z}\right)}{\partial z}=0
$$

where $t$ is time, $\rho$ is density, $u_{x}$ is the velocity component in the $x$ direction, $u_{y}$ is the velocity component in the $y$ direction, and $u_{z}$ is the velocity component in the $z$ direction. In an uncompressed fluid, $\rho$ is constant and Eq. (2) can be rewritten as 


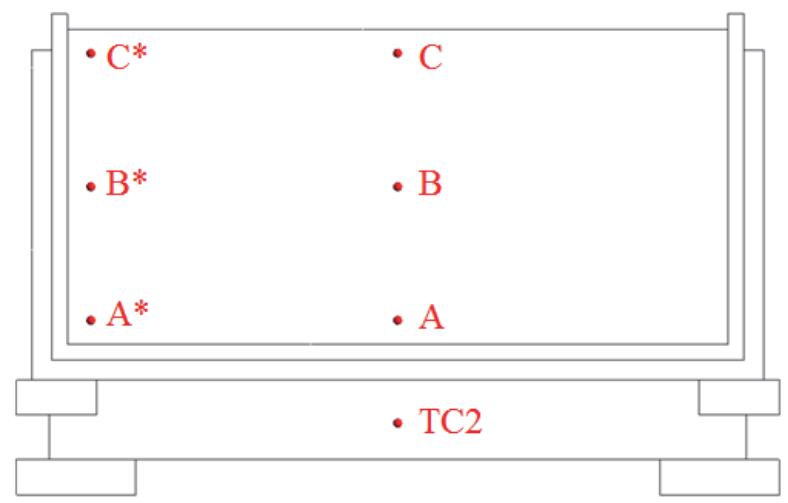

Fig. 4. (Color online) Locations of points where temperature changes were observed.

$$
\frac{\partial\left(\rho u_{x}\right)}{\partial x}+\frac{\partial\left(\rho u_{y}\right)}{\partial y}+\frac{\partial\left(\rho u_{z}\right)}{\partial z}=0
$$

When the Hamiltonian differential operator is introduced into Eq. (3), the equation becomes

$$
\nabla=i \frac{\partial}{\partial x}+j \frac{\partial}{\partial y}+k \frac{\partial}{\partial z}
$$

which can also be expressed as

$$
\nabla \cdot(\rho \vec{u})=0
$$

Next, the momentum equations are as follows:

$$
\begin{aligned}
& \frac{\partial\left(\rho u_{x}\right)}{\partial t}+\nabla \cdot\left(\rho u_{x} \vec{u}\right)=-\frac{\partial P}{\partial x}+\frac{\partial \tau_{x x}}{\partial x}+\frac{\partial \tau_{y x}}{\partial y}+\frac{\partial \tau_{z x}}{\partial z}+\rho f_{x}, \\
& \frac{\partial\left(\rho u_{y}\right)}{\partial t}+\nabla \cdot\left(\rho u_{y} \vec{u}\right)=-\frac{\partial P}{\partial y}+\frac{\partial \tau_{x y}}{\partial x}+\frac{\partial \tau_{y y}}{\partial y}+\frac{\partial \tau_{z y}}{\partial z}+\rho f_{y}, \\
& \frac{\partial\left(\rho u_{z}\right)}{\partial t}+\nabla \cdot\left(\rho u_{z} \vec{u}\right)=-\frac{\partial P}{\partial z}+\frac{\partial \tau_{x z}}{\partial x}+\frac{\partial \tau_{y z}}{\partial y}+\frac{\partial \tau_{z z}}{\partial z}+\rho f_{x},
\end{aligned}
$$

where $P$ is the static pressure, $\tau$ is the component of shear stress, and $f$ is the mass force. If the momentum is only affected by the gravity force and the $y$-axis is vertically downward, then $f_{x}=f_{z}=0$ and $f_{y}=g$. The energy equations can be expressed as 


$$
\begin{aligned}
\frac{\partial(\rho E)}{\partial t}+\nabla \cdot[\vec{u}(\rho E+p)] & =\nabla \cdot\left[k_{e f f} \nabla T-\sum_{j} h_{j} J_{j}+\left(\tau_{e f f} \cdot \vec{u}\right)\right]+S, \\
E & =h-\frac{p}{\rho}+\frac{V^{2}}{2},
\end{aligned}
$$

where $\frac{\partial(\rho E)}{\partial t}, \nabla \cdot\{\vec{u}(\rho E+p)\}, k_{e f f} \nabla T, \sum_{j} h_{j} J_{j}, \quad\left(\tau_{e f f} \cdot \vec{u}\right)$, and $S$ depend on the time, convection, conduction, material diffusion, viscous dissipation, and enthalpy. $E$ is the total energy (internal energy + dynamic energy + static energy), $\vec{u}$ is the velocity vector, $h_{j}$ is the enthalpy, $J_{j}$ is the diffusion of the fluid, $k_{e f f}$ is the thermal conductivity, and $S$ is the heat source item.

Next, the turbulence equations ( $k-\varepsilon$ equations) are given by

$$
\begin{gathered}
\frac{\partial\left(\rho k_{1}\right)}{\partial t}+\frac{\partial\left(\rho k_{1} u_{i}\right)}{\partial x_{i}}=\frac{\partial}{\partial x_{j}}\left[\left(\mu+\frac{\mu_{t}}{\sigma_{k}}\right) \frac{\partial k_{1}}{\partial x_{j}}\right]+G_{k}+G_{b}-\rho \varepsilon_{1}-Y_{M}, \\
\frac{\partial\left(\rho \varepsilon_{1}\right)}{\partial t}+\frac{\partial\left(\rho \varepsilon_{1} u_{i}\right)}{\partial x_{i}}=\frac{\partial}{\partial x_{j}}\left[\left(\mu+\frac{\mu_{t}}{\sigma_{\varepsilon}}\right) \frac{\partial \varepsilon_{1}}{\partial x_{j}}\right]+\rho C_{1} E \varepsilon_{1}-\rho C_{2} \frac{\varepsilon^{2}}{k_{1}+\sqrt{v \varepsilon}}+C_{1 \varepsilon} \frac{\varepsilon_{1}}{k_{1}} C_{3 \varepsilon} G_{b},
\end{gathered}
$$

in which $C_{1}=\max \left(0.43, \frac{\eta}{\eta+5}\right), \quad \eta=\left(2 E_{i j} E_{i j}\right)^{\frac{1}{2}} \frac{k}{\varepsilon}, \quad E_{i j}=\frac{1}{2}\left(\frac{\partial u_{i}}{\partial x_{j}}+\frac{\partial u_{j}}{\partial x_{i}}\right), \quad \mu_{t}=\rho C_{\mu} \frac{k^{2}}{\varepsilon}$, $C_{\mu}=\frac{1}{A_{0}+A_{S} \frac{k U^{*}}{\varepsilon}}, \quad A_{S}=\sqrt{6} \cos \varphi, \quad \varphi=\frac{1}{3} \cos ^{-1}(\sqrt{6} W), \quad W=\frac{S_{i j} S_{j k} S_{k i}}{\tilde{S}^{3}}, \quad \tilde{S}=\sqrt{S_{i j} S_{i j}}$, $S_{i j}=\frac{1}{2}\left(\frac{\partial u_{j}}{\partial x_{i}}+\frac{\partial u_{i}}{\partial x_{j}}\right), U^{*}=\sqrt{E_{i j} E_{i j}+\ddot{\Omega}_{i j} \ddot{\Omega}_{i j}}, \ddot{\Omega}_{i j}=\Omega_{i j}-2 \varepsilon_{i j k} \varpi_{k}$, and $\Omega_{i j}=\bar{\Omega}_{i j}-\varepsilon_{i j k} \varpi_{k}$, where $k_{1}$ is the turbulent kinetic energy, $\varepsilon_{1}$ is the kinetic energy dissipation rate, $G_{k}$ is the turbulent kinetic energy generated by the average velocity gradient, $G_{b}$ is the turbulent kinetic energy generated by the buoyant force, $Y_{M}$ is the dissipation rate, $C_{1 \varepsilon}$ is 1.44 (empirical constant), $C_{3 \varepsilon}$ is 0.09 (empirical constant), $C_{2}$ is 1.9 (empirical constant), $A_{0}$ is 4.0 (empirical constant), $\sigma_{k}$ is 1.0 (Planck constant of turbulence relative to turbulent kinetic energy), $\sigma_{\varepsilon}=1.3$ (Planck constant of turbulent dissipation rate), $\bar{\Omega}_{i j}$ is the rotation tensor, $\varpi_{k}$ is the angular velocity, $A_{0}$ and $A_{S}$ are constants, and $C_{\mu}$ is a function of the average strain and rotation rate. The radiation equation (DO equation) can be expressed as

$$
\nabla \cdot\{I(\vec{r}, \vec{s}) \vec{s}\}+\left(a+\sigma_{s}\right) I(\vec{r}, \vec{s})=a n^{2} \frac{\sigma T^{4}}{\pi}+\frac{\sigma_{s}}{4 \pi} \int_{0}^{4 \pi} I\left(\vec{r}, \vec{s}^{\prime}\right) \Phi\left(\vec{s} \cdot \vec{s}^{\prime}\right) d \Omega^{\prime},
$$


where $a$ is the gas absorption coefficient, $\sigma_{s}$ is the gas scattering coefficient, $I$ is the light intensity, $\vec{r}$ is the position vector, $\vec{s}$ is the direction vector, $\vec{s}^{\prime}$ is the scattering direction vector, $T$ is the temperature of the local gas, $\sigma$ is the Boltzmann constant $\left(5.669 \times 10^{-8} / \mathrm{m}^{2} \cdot \mathrm{K}^{7}\right), \Phi$ is the phase function, $\Omega^{\prime}$ is the solid angle, and $n$ is the wavelength. Finally, the energy equations of the solidification problem are

$$
\begin{aligned}
& \frac{\partial}{\partial t}(\rho H)+\nabla \cdot(\rho \vec{v} H)=\nabla \cdot(k \nabla T)+S, \\
& \square \square \quad \text { and } h=h_{r e f}+\int_{T_{r e f}}^{T} c_{p} d T,
\end{aligned}
$$

where $H$ is the enthalpy, $\vec{v}$ is the velocity vector of flow field, $\Delta H$ is the latent heat, $c_{p}$ is the specific heat, $h_{r e f}$ is the reference enthalpy, and $T_{r e f}$ is the reference temperature.

To show the difference between the simulation and actual conditions, Fig. 5 shows the measured and simulated temperature curves at point TC2. The error tolerance is about $6 \%$. This result demonstrates that the CFD software can be used to simulate the process of silicon ingot growth in the DSS furnace. Next, we used the variation of the slope to find the change in the solid-liquid interface shape. We used the lowest point as a benchmark, the section from the lowest point to the crucible-wall area was referred to as the wall-face section, and the highest point of the crucible-wall section was located on the crucible wall. Using the lowest and highest points, we calculated the average slope in the solid-liquid interface of the crucible-wall section, which we called the concavity, the section from the lowest point to the center of the crucible was referred to as the central section, and in the central section, the highest point of the solid-liquid interface was usually at the center of the molten silicon. The average slope of the solid-liquid central section calculated from the highest and lowest points was referred to as the convexity. The concavity was defined as a negative value, and the smaller its value, the more concave the

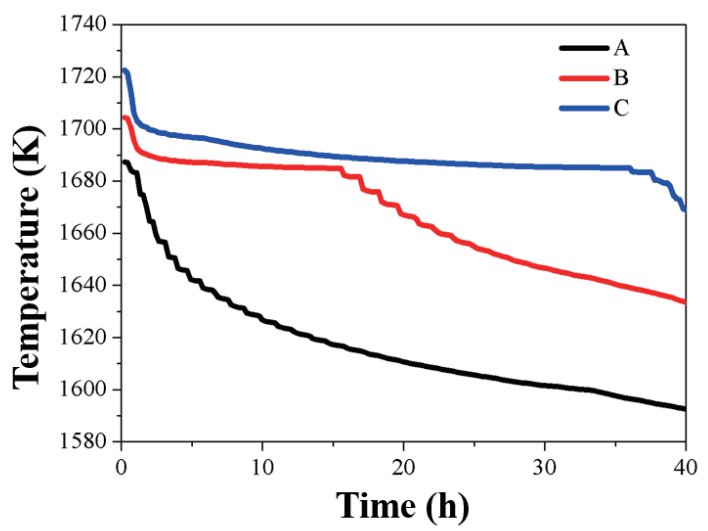

(a)

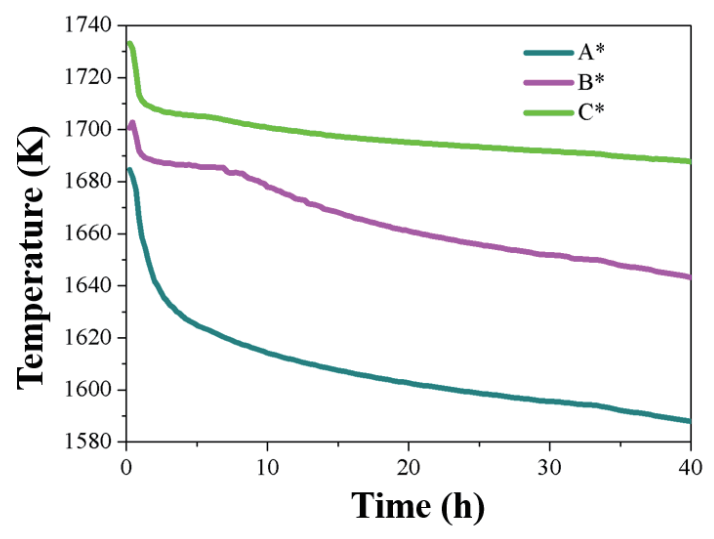

(b)

Fig. 5. (Color online) (a) Temperature-time curves of points A, B, and C, (b) temperature-time curves of points $\mathrm{A}^{*}, \mathrm{~B}^{*}$, and $\mathrm{C}^{*}$. 
solid-liquid interface of the crucible-wall section. The convexity was defined as a positive value, and the larger its value, and the more convex the solid-liquid interface of the central section. The concavity and convexity are expressed as

$$
\text { Concavity }=-\frac{d y_{2}}{d x_{2}} \text { and Convexity }=\frac{d y_{1}}{d x_{1}}
$$

Figures 5(a) and 5(b) show the simulation time-temperature curves of the points shown in Fig. 4, where the vertical spacing between each line is the temperature difference between different points. A larger spacing indicates that the vertical temperature gradient is higher, meaning that the DSS furnace is more able to drive the growth of the crystal silicon vertically upwards. Among points A, B, and C, point $\mathrm{C}$, at the highest location and nearest the heater, had the lowest cooling rate, and $36 \mathrm{~h}$ was required to completely release the latent heat. Point $\mathrm{A}$ had the lowest location and the highest cooling rate, and only $0.6 \mathrm{~h}$ was required to completely release the latent heat. Therefore, the growth rate of the silicon ingot was highest at the beginning, then slowly decreased. Points $\mathrm{A}^{*}, \mathrm{~B}^{*}$, and $\mathrm{C}^{*}$ were closer to the crucible-wall section than points $\mathrm{A}, \mathrm{B}$, and $\mathrm{C}$, but their cooling rates were higher than those of points $\mathrm{A}, \mathrm{B}$, and $\mathrm{C}$ in the central section of the molten silicon.

Figure 6(a) shows the temperature difference curve between points $\mathrm{A}$ and $\mathrm{C}$, which is the axial temperature change. When the silicon at point $\mathrm{A}$ solidified, point $\mathrm{C}$ had a high temperature of $1685 \mathrm{~K}$, and the silicon at this point was still molten. The heat transfer characteristic of the solidified silicon was better than that of the molten silicon, and the silicon at the bottom of the furnace had the fastest heat dissipation. The temperature difference between points $\mathrm{A}$ and $\mathrm{C}$ increased gradually until the silicon at point $\mathrm{C}$ was solidified. As shown in Fig. 6(a), the maximum temperature difference between points $\mathrm{A}$ and $\mathrm{C}$ was $88.41 \mathrm{~K}$. Because the silicon at point $\mathrm{C}$ was solidified after $36 \mathrm{~h}$, the temperature decreased gradually.

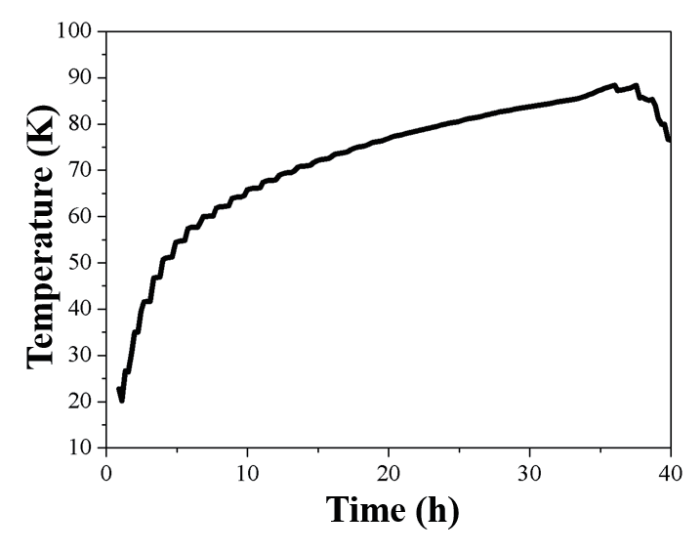

(a)

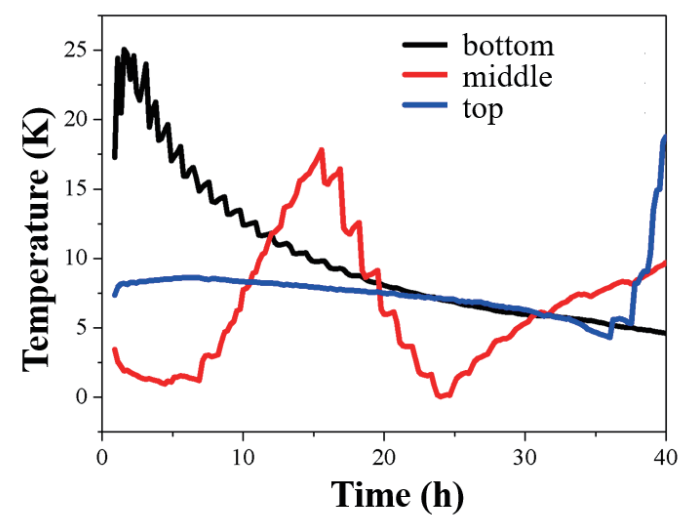

(b)

Fig. 6. (Color online) (a) Temperature difference curve between points $\mathrm{A}$ and $\mathrm{C}$ and (b) temperature difference between each point in the central section and the corresponding point in the crucible-wall section. 
Figure 6(b) shows the difference in the radial temperature between each point in the central section and the corresponding point in the crucible-wall section. The change in the radial temperature in the top area was because the Ar gas flow had a strong influence on temperature gradient, and the silicon at point $\mathrm{C}$ was solidified first. Point $\mathrm{C}^{*}$ was closer to the heater than point $\mathrm{C}$, and its temperature remained at $1685 \mathrm{~K}$; thus, the temperature difference between the two points increased with time. The difference in the central radial temperature resulted in the silicon at point $\mathrm{B}^{*}$ being solidified at $7 \mathrm{~h}$ and the silicon at point $\mathrm{B}$ being solidified at $14 \mathrm{~h}$, and the maximum temperature difference between the two points was $17.84 \mathrm{~K}$. When the silicon at point $\mathrm{B}$ was solidified, the difference in the radial temperature dropped. The difference in the central radial temperature at the bottom meant that the silicon at point $\mathrm{A}^{*}$ was instantly solidified at the beginning of growth and the silicon at point A was solidified at $0.6 \mathrm{~h}$, then the difference in the radial temperature dropped gradually.

Diagrams showing the variation of the temperature profile as a function of cooling time are shown in Fig. 7. As the cooling time increased from 10 to $40 \mathrm{~h}$, it can be clearly seen that the
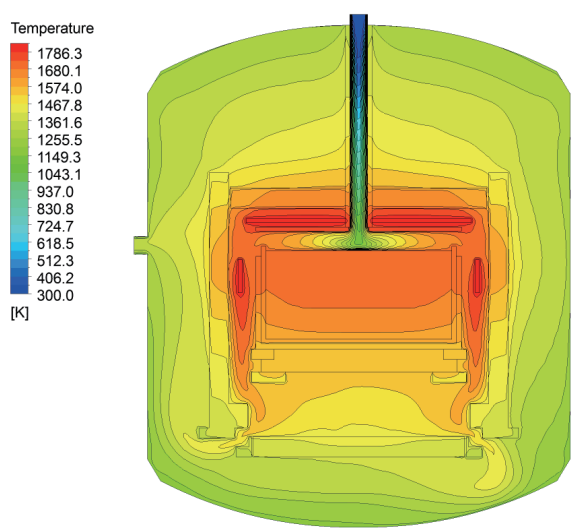

(a)

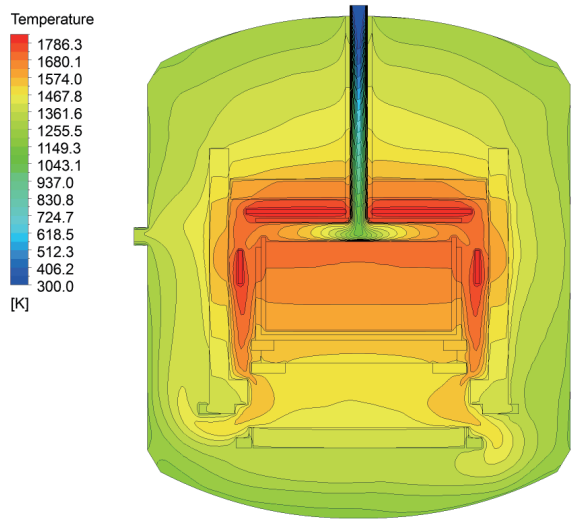

(c)

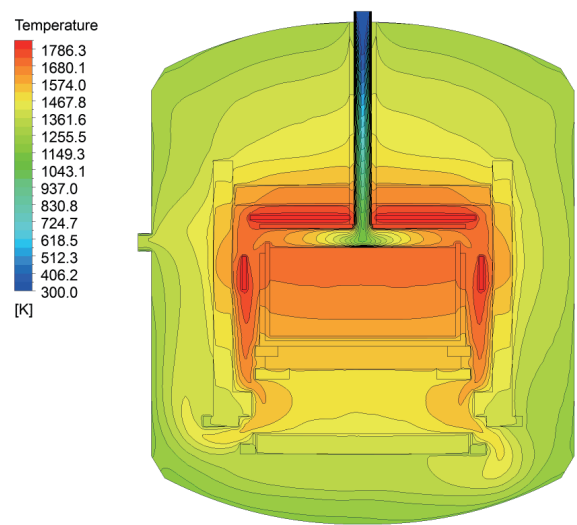

(b)

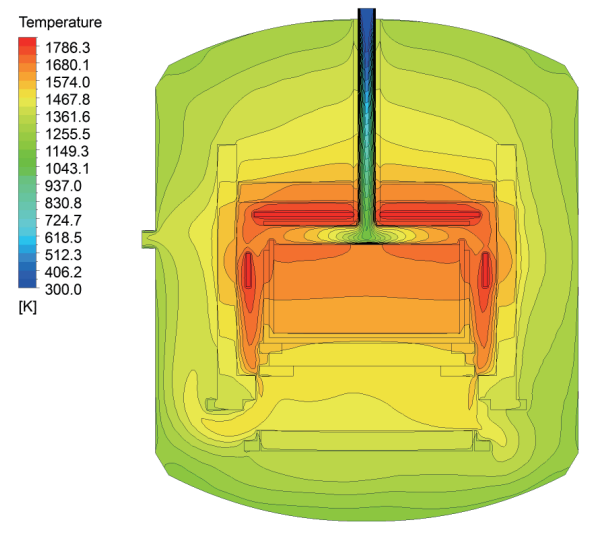

(d)

Fig. 7. (Color online) Simulation results of temperature profiles at (a) $10 \mathrm{~h}$, (b) $20 \mathrm{~h}$, (c) $30 \mathrm{~h}$, and (d) $40 \mathrm{~h}$. 
bottom of the silicon gradually cooled. The thermal energy escaped from the bottom clearance of the graphite insulating plate, and the radial temperature gradient of the silicon material in the crucible was proportional to the distance from the thermal heater. Figure 8 shows the simulated solid-liquid phase distribution map, where the cyan zone was the region of $100 \%$ solid silicon, the red zone was the region of $100 \%$ liquid silicon, and the zone between the cyan and red zones was the solid-liquid fuzzy zone. To extract the required points with the lowest concavity and convexity, the area of the 50\% fuzzy zone was defined as the solid-liquid interface.

Figure 9 shows the solid-liquid phase distribution maps at different times. Because the crucible side walls cooled fastest, the silicon ingot started to grow from the two side walls. If the radial temperature gradient can be well controlled to ensure that it is not too large, we can prevent the silicon ingot from starting to grow from the side walls. If the silicon ingot grows from the side walls, a long-strip-type crystal lattice will form. Such a crystal lattice would cause the dopants to accumulate in the grain boundaries, and the higher impurity concentration would degenerate the energy conversion efficiency of the grown silicon wafer.

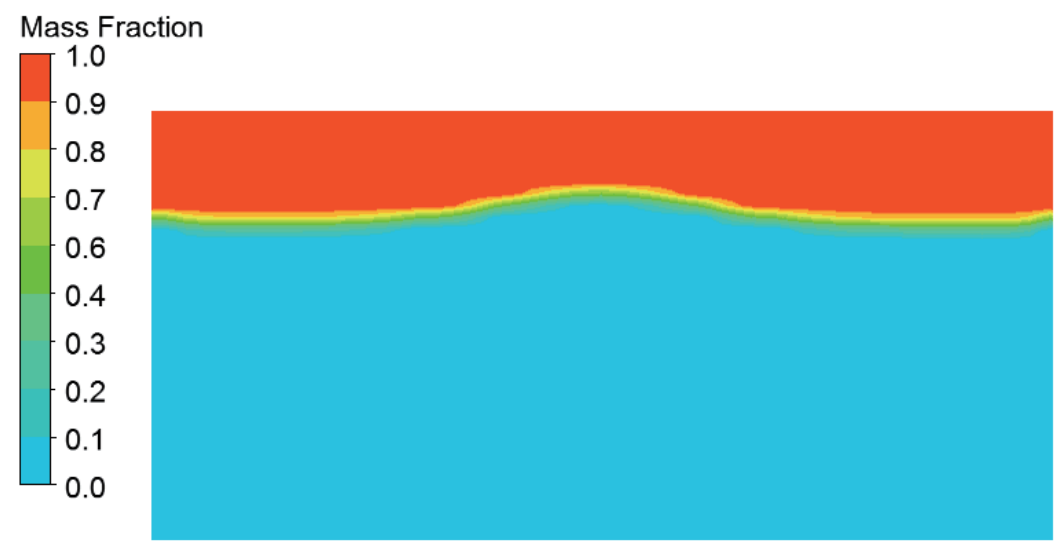

Fig. 8. (Color online) Solid-liquid phase distribution map.

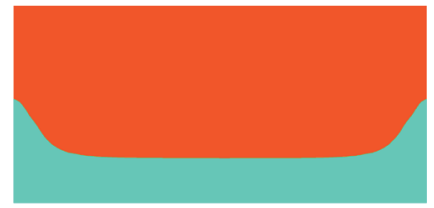

(a)

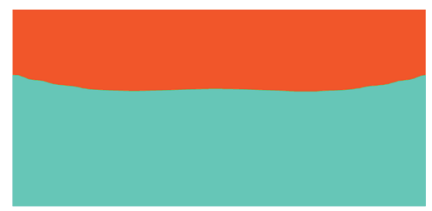

(d)

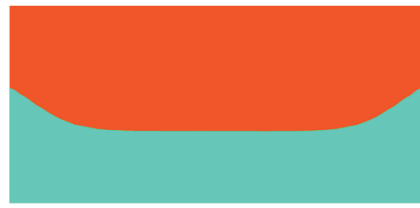

(b)

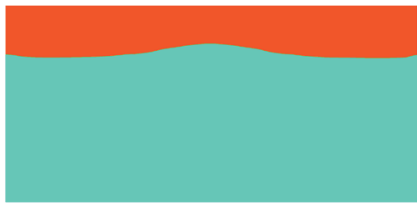

(e)

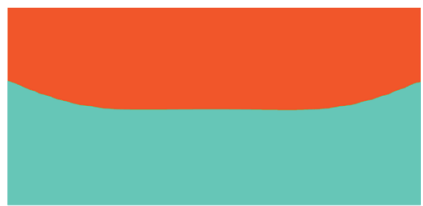

(c)

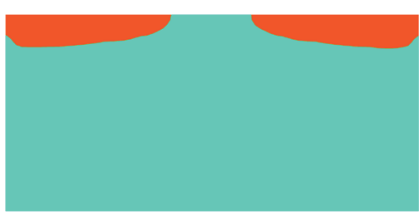

(f)

Fig. 9. (Color online) Solid-liquid phase distribution maps after cooling times of (a) $5 \mathrm{~h}$, (b) $10 \mathrm{~h}$, (c) $15 \mathrm{~h}$, (d) $20 \mathrm{~h}$, (e) $30 \mathrm{~h}$, and (f) $40 \mathrm{~h}$. 


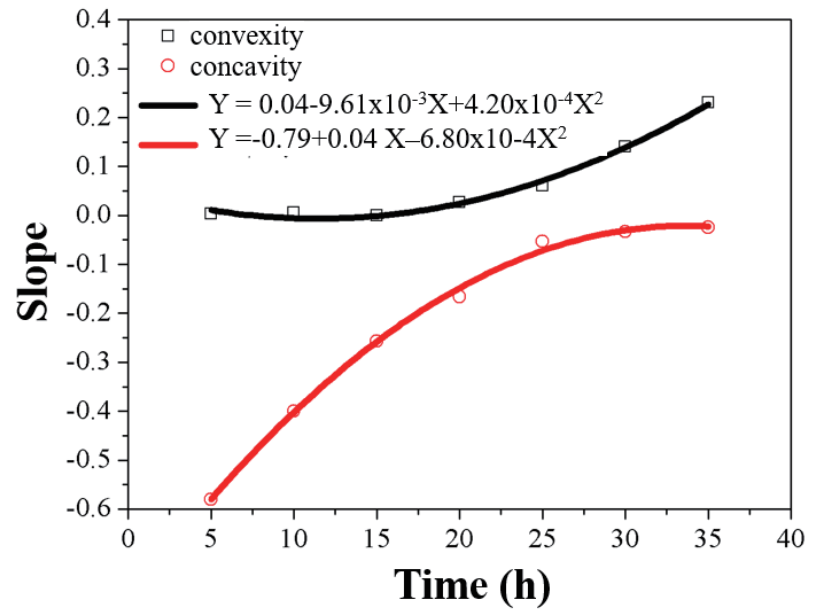

Fig. 10. (Color online) Convexity and concavity obtained from the simulation results.

Figure 10 shows the variations of concavity and convexity values as a function of time, which can be fitted as Eq. (17) and (18), respectively.

$$
\begin{gathered}
Y=0.04-9.61 \times 10^{-3} X+4.20 \times 10^{-4} X^{2} \\
Y=-0.79+0.04 X-6.80 \times 10^{-4} X^{2}
\end{gathered}
$$

As shown in Fig. 9, as the cooling time increased from 5 to $15 \mathrm{~h}$, the solid-liquid interface changed from flat to convex, the convexity increased, and the solid silicon had a convex outward appearance. With increasing cooling time, the crystal grains of the central section grew more outward, and the growth direction of the silicon ingot was not sufficiently vertical. As shown in Fig. 10, the maximum convexity was 0.23 and the minimum concavity was -0.02 . These simulation results have demonstrated that we can use CFD software to simulate the growth conditions of a silicon ingot in a DSS furnace. We believe that simulation technology can optimize the growth parameters of the directional solidification process for growing polycrystalline silicon ingots with higher efficiency.

\section{Conclusions}

In our simulation of crystal growth in a DSS furnace, we used triangle unstructured meshes for the unmovable region and quadrilateral structured meshes for the other parts and the fluid field. The continuity equation, the momentum equations, the energy equations, the turbulence equations, and the radiation equation were successfully used to simulate the growth of a silicon ingot in a DSS furnace. We found that the maximum temperature difference between points $\mathrm{B}$ and $\mathrm{B}^{*}$ was $17.84 \mathrm{~K}$. From the simulation results, we also found that as the cooling time increased from 5 to $35 \mathrm{~h}$, the maximum convexity was 0.23 and the minimum concavity was -0.02 . These 
results demonstrated that the simulation technology can optimize the growth parameters of the directional solidification process for growing polycrystalline silicon ingots with higher efficiency.

\section{Acknowledgments}

This work was supported by project numbers MOST 108-2221-E-390-005 and MOST 1092221-E-390-023.

\section{References}

1 F. Dughiero, M. Forzan, D. Ciscato, and F. Giusto: 2011 37th IEEE Photovoltaic Specialists Con., Seattle, WA, USA (2011) 002151.

2 X. Ma, L. Zheng, H. Zhang, B. Zhao, C. Wang, and F. H. Xu: J. Cryst. Growth 318 (2011) 288.

3 Y. T. Wong, C. Hsu, and C. W. Lan: J. Cryst. Growth 387 (2014) 10.

4 S. Kasap and P. Capper: Springer Handbook of Electronic and Photonic Materials (Springer, Cham, 2017) 2nd ed.

5 G. Fisher, M. R. Seacrist, and R. W. Standley: Proc. IEEE Special Centennial Issue (2012) 1454-1474.

6 J. W. Shur, B. K. Kang, S. J. Moon, W. W. So, and D. H. Yoon: Sol. Energy Mater. Sol. Cells 95 (2011) 3159.

7 H. L. Zhang, D. You, C. L. Huang, Y. H. Wu, Y. Xu, and P. Wu: J. Cryst. Growth 435 (2016) 91.

8 Y. Y. Yu, J. J. Ding, W. L. Chen, Z. Y. Zhang, X. C. Zhou, G. X. Zhong, and X. M. Huang: J. Mater. Sci. Eng. B 6 (2016) 304.

9 Silicon: Evolution and Future of a Technology, Cha. 20, Silicon Sensors, P. Siffert and E. F. Krimmel, Eds. (Springer, Berlin 2004).

10 R. F. Balderas-Valadez, V. Agarwala, and C. Pacholski: RSC Adv. 6 (2016) 21430.

11 J. W. Shur, B. K. Kang, S. J. Moon, W. W. So, and D. H. Yoon: Sol. Energy Mater. Sol. Cells 95 (2011) 3159.

12 M. P. Bellmann, B. Panjwani, M. Syvertsen, and E. A. Meese: J. Cryst. Growth 369 (2013) 47.

13 A. Popescu and D. Vizman: Inter. J. Heat Mass Transfer 54 (2011) 5540.

14 L. Chen and B. Dai: J. Cryst. Growth 354 (2012) 86. 